\title{
Chair Based Measurements of Sitting Behavior a Field Study of Sitting Postures and Sitting Time in Office Work
}

\author{
Matthijs P. Netten ${ }^{1}$, L.H.M. van der Doelen ${ }^{1,2}$, and Richard H.M. Goossens ${ }^{1,3}$ \\ ${ }^{1}$ Delft University of Technology, Faculty of Industrial Design Engineering, Section Applied \\ Ergonomics and Design, Delft, The Netherlands \\ $\{\mathrm{m}$.p.netten, R.H.M.Goossens $\}$ atudelft.nl \\ ${ }^{2}$ BMA Ergonomics, Zwolle, The Netherlands \\ B.van.der.doelen@bma-ergonomics.com \\ ${ }^{3}$ Erasmus University Rotterdam, Department of Neuroscience, The Netherlands
}

\begin{abstract}
In order to understand the relation between prolonged sitting and the relation to health outcome, the behavior while being seated must be studied.

A total of 41 office workers participated in a study whilst performing their regular work for eight weeks, whilst sitting on a measuring office chair (Smart Chair). The first two (control) weeks they were not aware of the measuring abilities of the chair. After this, two groups were made to distinguish between the effects of chair instruction and smart feedback on sitting postures (Van der Doelen et al. 2011).

In this paper the data has been analyzed in another way. The aim of this paper is to explore the characteristics of sedentary behavior for 41 subjects during their regular office work over eight weeks by measuring the events of sitting and absence from their office chair.

Results showed that the office workers in this study on average have very long sitting events, that exceed general recommendations. Results showed that the office workers in this study on average have very long sitting events, that exceed general recommendations. Recommendations for 5 minute breaks every hour are met by $85 \%$ of the participants. However recommendations on sitting les than 20 minutes were met by $5 \%$ of the participants. None of the participants met the recommendations on all of their days during the field study.

The sedentary behavior shown in this study underlines the importance to monitor and influence sedentary behavior while considering the individual sedentary patterns. Further knowledge on analyzing sedentary patterns is needed.
\end{abstract}

Keywords: device-based measures, sedentary behavior.

\section{Introduction}

The amount of time spent in sedentary situations has increased since the introduction of new communication technologies, like the computer. Some studies report that prolonged sitting can have adverse effects on health outcomes [1]. For some people the workplace covers an important part of the sedentary life of people and it is 
suggested that for those workers it is the key setting for health promotion interventions [2]. Therefore, in order to understand the relation between prolonged sitting and the relation to health outcome, the behavior while being seated at the office must be studied.

Self-reported measures for sedentary behavior are used [3] or device-based measures [2,4,5]. Although research suggests that self-reported measures are a valid method to assess sedentary behavior [6],it is also noted that this method cannot uncover the more detailed patterns of sitting during the day [6]. Device-based measures overcome this problem. Most device-based measures use accelerometer technology $[2,4,5]$. Sedentary behavior can be studied in more detail and has shown e.g. that workdays are associated with more sitting and less walking/standing time than leisure days [2].

Another approach is to place sensors in the office chair that can measure presence or even postures $[7,8,9,10]$. The current research utilized such a device-based measuring tool that is not noticeable to the respondent. The office chair in our study, has integrated sensors that can both measure time spent sitting as well as calculate sitting postures. The chair can give feedback to the end-user by means of a tactile signal and a label attached to the chair. This, so called Smart Chair has been developed and launched by the Dutch company BMA Ergonomics.

The aim of this paper is to explore the characteristics of sedentary behavior for 41 subjects during their regular office work over eight weeks by measuring the events of sitting and absence from their office chair.

\section{Method}

A field study using office chairs with measuring technology was conducted from October to December 2010. Subjects were employees of a large financial institute in Brussels, Belgium. They could be included in the study if they worked at least 3 days a week and reported working with a computer more than 4 hours per day. They were not included if there was a history of musculoskeletal problems in the last 6 months.

\section{$2.1 \quad$ Subjects}

Participants were 41 volunteers (19 females, 22 males) that worked at four different departments. The participants were divided into two research groups, each consisting of two occupational groups; 'Administrative' and 'IT'. The participants should perform their regular office tasks while using a measuring chair.

Female participants had a mean age of 42.4 yrs (sd 8.6). They had a mean height of $166.1 \mathrm{~cm}(\mathrm{sd} 5.9)$ and a mean body weight of $70.0 \mathrm{~kg}$ ( $\mathrm{sd} 12.2)$. Male participants had a mean age of $46.3 \mathrm{yrs}$ (sd 9.9), a mean height of $179.0 \mathrm{~cm}(\mathrm{sd} \mathrm{6.2)}$ and a mean weight of $84.2 \mathrm{~kg}$ (sd 11.3). 


\subsection{Measuring (Office) Chair: Smart Chair}

The chair calculates postures based on pressure values of sensors in the seat surface and back of the seat. Postures are calculated at $1 \mathrm{~Hz}$ and logged by the chair. The measuring system is fully integrated in the chair cushioning. Figure 1 shows the different postures that the Smart Chair can distinguish. In this paper we only report the time that participants were sitting on the chair in any posture versus the time that the participant was absent from the chair.

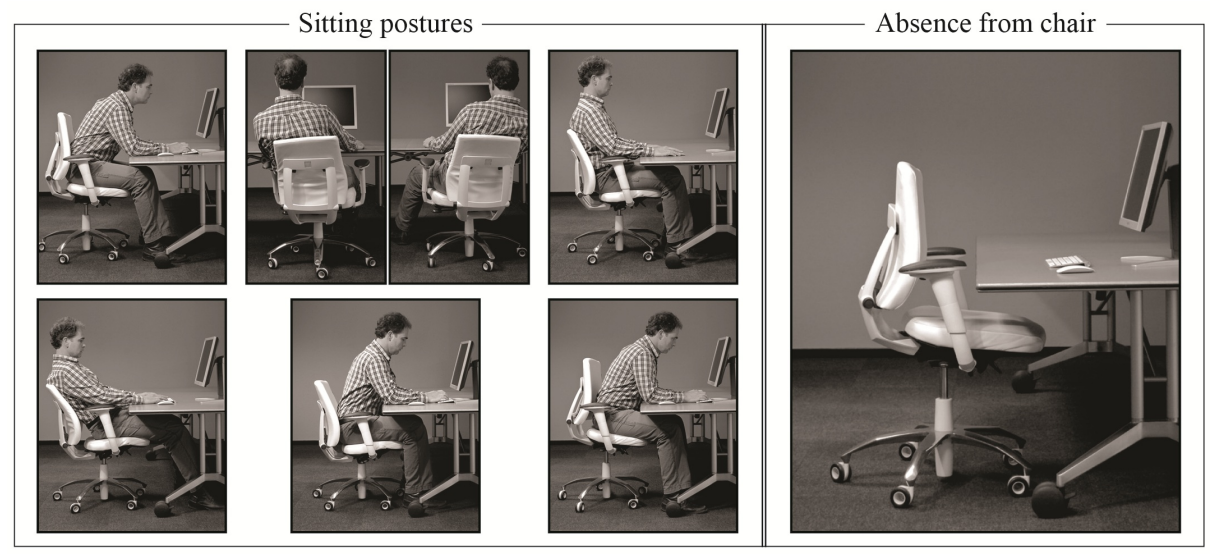

Fig. 1. Postures by the Smart Chair

\subsection{Experimental Set-up}

Subjects used the chairs during 8 weeks. The first two weeks were considered a control condition to measure regular sitting behavior. During these first two weeks the subjects received a Smart Chair that measured their posture, but no instruction or feedback was given during that time.

At the start of the intervention condition all participants received the same training instruction about chair settings and ergonomic workplace settings (e.g monitor height, desk height). Part of them ( $n=22)$ also got extra feedback from the chair about sitting behavior [10].

The researchers collected the data from the chairs at times that the users were absent.

\subsection{Data Analysis}

Duration of sitting events, duration of absence events, number of sitting events per workday, number of absence events per workday and the total time of the workday were calculated from the data for each subject. Figure 2 illustrates these measures. The length of a workday is defined as the time between the first contact with the chair 


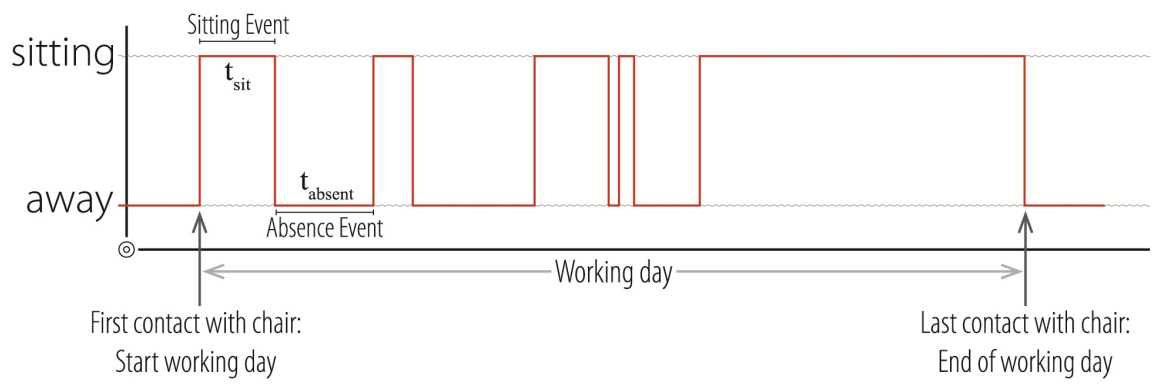

Fig. 2. Outcome measurements in Sitting pattern

on that day and the last contact with the chair on that day. A sitting event is defined as the interval where the chair measures postures (presence). As soon as the chair measures no postures for more than 60 seconds, the absence event starts.

The calculated measures were averaged according to the subgroups that were defined in the original study (experimental groups A and B, administrative versus IT and male versus female participants), to explore potential differences. Statistical comparisons were not made.

Adherence to sitting recommendations was assessed using three different boundaries. Ryan et al (2011) reported these from general recommendations by experts. He concluded that these guidelines still lack a scientific validation, but they deliver do insights in how the different recommendations are met.

The recommendations used were a maximum sitting time of $20 \mathrm{~min}$; a maximum sitting time of $30 \mathrm{~min}$ and a 5-min break every hour, which was operationalized as a maximum sitting time of $55 \mathrm{~min}$.

\section{Results}

For the 41 subjects a total of 964 working days were collected that met the criteria. The study was performed with a Smart Chair prototype that was still in development. Some data was lost due to technical issues. This led to a loss of 134 working days.

\subsection{Typical Data}

Lengths of the sitting events and absence events were used to depict (figure 3) sitting patterns from three randomly selected participants.

Participant 2 remains sedentary ( $84,8 \%$ of the working day with 6 sitting events of $74 \pm 46 \mathrm{~min}$ ) longer than participant 1 (18 sitting events of $16,1 \pm 21,3 \mathrm{~min}$ ) or participant 3(22 sitting events of 18,8 $\pm 18,5 \mathrm{~min})$. 

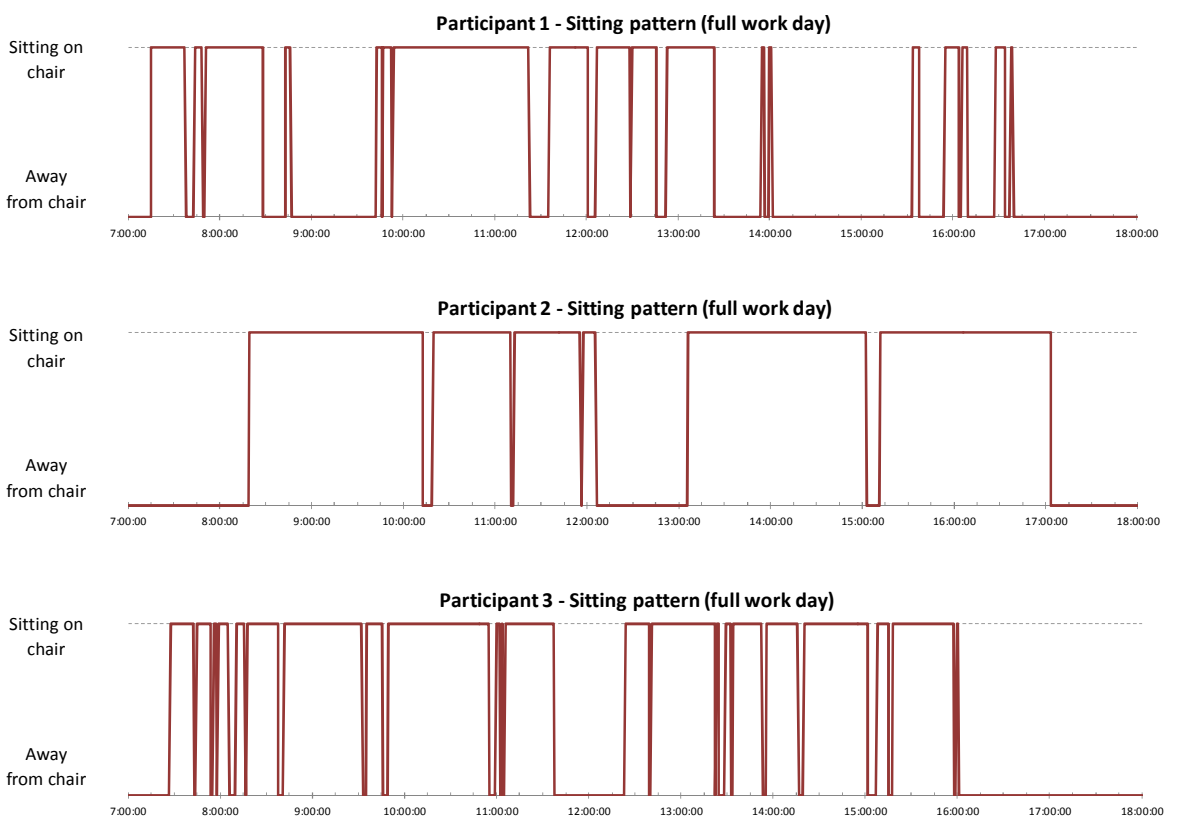

\begin{tabular}{|c|c|c|c|c|c|c|c|c|}
\hline \multirow[b]{2}{*}{ Participant 1} & \multirow{2}{*}{$\begin{array}{c}\begin{array}{c}\text { Working day } \\
\text { (hrs) }\end{array} \\
9,4 \\
\end{array}$} & \multirow{2}{*}{$\begin{array}{c}\begin{array}{c}\text { Total sitting time } \\
\text { (hrs) }\end{array} \\
4,8 \\
\end{array}$} & \multirow{2}{*}{$\begin{array}{c}\text { \% Workday } \\
51,2 \% \\
\end{array}$} & \multirow{2}{*}{$\begin{array}{c}\begin{array}{c}\text { Number of sitting } \\
\text { events }\end{array} \\
18 \\
\end{array}$} & \multirow{2}{*}{$\begin{array}{c}\begin{array}{c}\text { Longest Sitting } \\
\text { event (min) }\end{array} \\
89,0\end{array}$} & $\begin{array}{c}\text { Average time } \\
\text { sitting event (min) }\end{array}$ & \multicolumn{2}{|c|}{$\begin{array}{c}\text { Average time } \\
\text { absence event } \\
\text { (min) }\end{array}$} \\
\hline & & & & & & $16,1 \quad \pm 21,3$ & 16,2 & $\pm 24,1$ \\
\hline Participant 2 & 8,7 & 7,4 & $84,8 \%$ & 6 & 116,4 & $74,2 \quad \pm 45,8$ & 15,9 & $\pm 24,9$ \\
\hline Participant 3 & 8,6 & 6,9 & $80,5 \%$ & 22 & 65,9 & $18,8 \pm 18,5$ & 4,8 & $\pm 9,7$ \\
\hline
\end{tabular}

Fig. 3. Typical data for three randomly chosen participants

\subsection{Sitting versus Absence}

Table 1. Sitting and absence events and their outcome divided in three different subgroups

\begin{tabular}{|c|c|c|c|c|c|c|c|c|c|c|c|c|}
\hline \multirow[b]{2}{*}{$\begin{array}{l}\text { Subgroup } \\
\quad \text { Research Phase }\end{array}$} & \multicolumn{2}{|c|}{$\begin{array}{l}\text { Working day } \\
\text { total time }\end{array}$} & \multicolumn{2}{|c|}{$\begin{array}{l}\text { Sitting time on } \\
\text { working days }\end{array}$} & \multicolumn{4}{|c|}{ Absence Eventes } & \multicolumn{4}{|c|}{ Sitting Events } \\
\hline & $\begin{array}{c}\text { Average } \\
\text { (hrs) }\end{array}$ & $\begin{array}{c}\text { sd } \\
\text { (hrs) }\end{array}$ & $\begin{array}{c}\text { Average } \\
\text { (hrs) }\end{array}$ & $\begin{array}{c}\% \text { of } \\
\text { workday }\end{array}$ & $\begin{array}{c}\text { Average } \\
(\mathrm{min})\end{array}$ & $\begin{array}{c}\mathrm{sd} \\
(\mathrm{min})\end{array}$ & $\begin{array}{c}\operatorname{Max} \\
(\min )\end{array}$ & $\begin{array}{l}\text { avg. events } \\
\text { / workday }\end{array}$ & $\begin{array}{c}\text { Average } \\
(\mathrm{min})\end{array}$ & sd $(\mathrm{min})$ & $\begin{array}{r}\operatorname{Max} \\
(\min )\end{array}$ & $\begin{array}{l}\text { avg. events } \\
\text { / workday }\end{array}$ \\
\hline Control condition & 8,04 & 1,44 & 5,22 & $64,8 \%$ & 14,1 & 24,9 & 253,8 & 12,1 & 24,0 & 25,6 & 181,7 & 13,1 \\
\hline Test condition & 7,98 & 1,23 & 5,08 & $63,6 \%$ & 13,4 & 26,7 & 388,3 & 13,0 & 21,7 & 24,1 & 208,7 & 14,0 \\
\hline \multicolumn{13}{|l|}{ B } \\
\hline \multicolumn{13}{|l|}{ Admin } \\
\hline \multirow{2}{*}{$\begin{array}{l}\text { Control condition } \\
\text { Test condition }\end{array}$} & 7,77 & 1,51 & 5,55 & $71,4 \%$ & 12,1 & 24,7 & 257,1 & 11,0 & 27,7 & 29,4 & 206,1 & 12,0 \\
\hline & 7,72 & 1,56 & 5,20 & $67,3 \%$ & 12,2 & 25,0 & 297,9 & 12,4 & 23,2 & 25,2 & 200,4 & 13,4 \\
\hline \multicolumn{13}{|l|}{ ICT } \\
\hline \multicolumn{13}{|l|}{ Female } \\
\hline Control condition & 8,12 & 1,12 & 5,70 & $70,2 \%$ & 14,1 & 25,4 & 207,2 & 10,3 & 30,3 & 32,1 & 181,7 & 11,3 \\
\hline Test condition & 7,96 & 1,37 & 5,52 & $69,4 \%$ & 13,2 & 25,0 & 257,1 & 11,0 & 27,6 & 29,8 & 206,1 & 12,0 \\
\hline \multicolumn{13}{|l|}{ Male } \\
\hline Control condition & 7,92 & 1,36 & 5,36 & $67,6 \%$ & 13,5 & 25,1 & 388,3 & 11,4 & 26,0 & 26,5 & 208,7 & 12,4 \\
\hline Test condition & 7,77 & 1,52 & 5,05 & $65,1 \%$ & 13,5 & 26,9 & 339,2 & 12,1 & 23,2 & 24,5 & 193,9 & 13,1 \\
\hline
\end{tabular}




\subsection{Adherence to Sitting Recommendations}

Table 2 shows that 5 minute breaks in 1 hour is attainable for $85 \%$ of the participants. However none of them met this recommendation on all their measured working days. $21 \pm 15 \%$ to $23 \% \pm 18 \%$ of the sitting events are longer than 55 minutes.

The 20 minute recommendation shows percentages of $5 \%$ of participant that meet this guideline. $53 \% \pm 20 \%$ to $49 \pm 20 \%$ of the sitting events are longer than 20 minutes.

Table 2. Adherence to sedentary guidelines for both test conditions

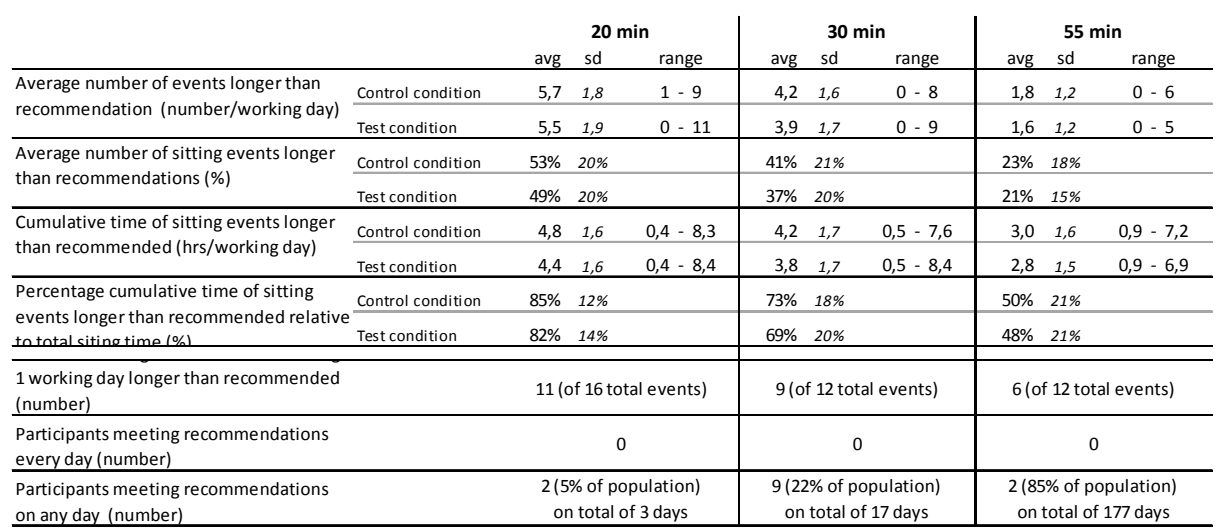

\section{Discussion}

The smart chair is used as an unobtrusive instrument to monitor behavior close to the workplace. This means it is not able to monitor individual sitting time on other chairs, e.g. during meetings or (lunch)breaks. Long absence events in chair data during lunch or meetings can be sedentary after all. However these breaks do count to interrupt sitting time. A workday in this study was defined as the time between first contact and last contact with the chair by the test user. Activities before or after this timeframe could be missing in the presented data. Total sitting time during workdays in this study can thus be underestimated, although this is expected to be a minor difference considering the average time of the observed working days that remains within a range of 7,7 - 8,2 hrs in subgroups.

McRady \& Levine (2009) reported total sitting times during workin days of 9,95 \pm $2 \mathrm{hr}$ using person bound accelerometers. Ryan (2011) found similar sitting times and absence times as found in this study.

The adherence to sedentary guidelines also showed similar findings. Although the participants of this study show even less adherence to the 20 minute guideline. Of the sitting events $49 \% \pm 20 \%-53 \% \pm 20 \%$ are longer than 20 minutes. These sitting times accumulate to $85 \% \pm 12 \%-82 \% \pm 14 \%$ of total sitting time. 
This study does show that the 5 minute break per hour seems attainable for most people. Although this study didn't show any participants that met recommendations during every day during the field study.

Participants were selected on their type of work (desk work, working minimum 4 hrs behind computer screen) which can partly explain the high total sitting time compared to other studies. The culture of work within the company that we visited can also be of influence.

Nonetheless the participants of this study show that many hours at work are spend sedentary. It must be noted that the individual differences can be large, as can be seen in figure 3. A measuring chair could therefor be a good solution to monitor and influence people in their sedentary behavior while considering these large individual differences.

All participants had a fixed personal workspace (desk and chair). Data was analyzed when it corresponded to their working schedules. All other data that was collected (coincidentally) by the chair was ignored for analysis.

The Smart Chairs that were used still were at a developing stage. Some technical problems were encountered. It did however result in missing data. It is assumed that the loss of data was a random event and not related to the outcome of sitting patterns per individual.

The study that was done, was not designed to explore sedentary behavior. The hypothesis underlying this data was whether the Smart Chair is able to influence sitting postures through feedback about sitting behavior .

Next step that are currently explored are about constructing a measure that can relate break regime (number of moments and timing) to sedentary events. The Smart Chair itself could be used to give feedback on exceeding sitting time recommendations directly on occurring of the event. At this point the system is mainly focussed on improving sitting postures.

Following the exploration of this data further analysis / research is wanted to gain more insights in the sitting patterns of office workers. With intensive office-base deskwork, measurements of sedentary times and times of absence with a measuring chair a good solution. This chair can deal with individual differences based on their sedentary patterns. Important aspects that need further study:

- Can longer sitting events be related with longer absence as compensation? Frequency distribution of these patterns can be considered to construct a risk index for sedentariness. Long sitting times with few moments to interrupt seem more high risk than similar total sitting time divided over more events. Discern the test subjects in degree of calmness. Calm versus restless sitter. And above this, can this be related to the amount and lengths of breaks/sitting time?

- Quality of sitting postures vs sitting patterns. Does number and length of brakes relate to the quality of postures during sitting bouts?

\section{Conclusion}

Results showed that the office workers in this study on average have very long sitting events, that exceed general recommendations. Recommendations for 5 minute breaks 
every hour are met by $85 \%$ of the participants. However recommendations on sitting les than 20 minutes were met by $5 \%$ of the participants. None of the participants met the recommendations on all of their days during the field study.

The sedentary behaviour shown in this study underlines the importance to monitor and influence sedentary behaviour while considering the individual sedentary patterns. Further knowledge on analyzing sedentary patterns is needed.

Acknowledgement.The researchers would like to thank the Belgian financial institute that enabled us to do field research on such a scale. The support and efforts during this research were and still are greatly appreciated.

Also, the support from the consortium member BMA Ergonomics (also the Belgian Subsidiary) and Salland Electronics during the research is greatly appreciated.

\section{References}

1. Thorp, A.A., Owen, N., Neuhaus, M., Dunstan, D.W.: Sedentary Behaviors and Subsequent Health Outcomes in Adults: A Systematic Review of Longitudinal Studies, 1996-2011. American Journal of Preventive Medicine 41(2), 207-215 (2011)

2. McCrady, S.K., Levine, J.A.: Sedentariness at work: How much do we really sit. Obesity 17(11), 2103-2105 (2009)

3. Jans, M.P., Proper, K.I., Hildebrandt, V.H.: Sedentary Behavior in Dutch Workers. Differences Between Occupations and Business Sectors. American Journal of Preventive Medicine 33(6), 450-454 (2007)

4. Chastin, S.F.M., Granat, M.H.: Methods for objective measure, quantification and analysis of sedentary behaviour and inactivity. Gait and Posture 31(1), 82-86 (2010)

5. Matthews, C.E., Chen, K.Y., Freedson, P.S., Buchowski, M.S., Beech, B.M., Pate, R.R., Troiano, R.P.: Amount of time spent in sedentary behaviors in the United States, 20032004. American Journal of Epidemiology 167(7), 875-881 (2008)

6. Clark, B.K., Thorp, A.A., Winkler, E.A.H., Gardiner, P.A., Healy, G.N., Owen, N., Dunstan, D.W.: Validity of self-reported measures of workplace sitting time and breaks in sitting time. Medicine and Science in Sports and Exercise 43(10), 1907-1912 (2011)

7. Tan, H.Z., Slivovsky, L.A., Pentland, A.P.: A Sensing Chair Using Pressure Distribution Sensors. IEEE/ASME Trans. Mechatronics 6(3), 261-268 (2001)

8. Mutlu, B., Krause, A., Forlizzi, J., Guestrin, C., Hodgins, J.K.: Robust, Low-Cost, NonIntrusive Recognition of Seated Postures. In: Proceedings of 20th ACM Symposium on User Interface Software and Technology (UIST 2007), Newport, RI (2007)

9. Zheng, Y., Morrell, J.B.: Avibrotactile feedback approach to posture guidance. In: 2010 IEEE Haptics Symposium, Waltham, Massachusetts, USA (Boston Area), pp. 351-358 (2010)

10. Van der Doelen, L.H.M., Netten, M.P., Goossens, R.H.M.: \&NES University of Oulu, Finnish Institute of Occupational Health, the Oulu University of Applied Sciences, University of Eastern Finland, and University of Lapland, E. R. Y, Tactile feedback to influence sitting behaviour during office work. In: äyrynen, S.V. (Ed.), NES - Wellbeing and Innovation Through Ergonomics, pp. 380-385. Oulu, Finland (2011)

11. Ryan, C.G., Grant, P.M., Dall, P.M., Granat, M.H.: Sitting patterns at work: Objective measurement of adherence to current recommendations. Ergonomics 54(6), 531-538 (2011) 